\title{
Development of MATLAB Code for m-ary PSK Constellation Diagram
}

\author{
Saed Thuneibat \\ Department of Electrical Engineering, Al-Balqa Applied University, Jordan
}

\begin{abstract}
The constellation diagram is a representation of a signals modulated by a digital passband modulation scheme It displays the signal as a two-dimensional X-Y plane scatter diagram in the complex plane at symbol sampling instants. In a more abstract sense, it represents the possible symbols that may be selected by a given modulation scheme as points in the complex plane. Measured constellation diagrams can be used to recognize the type of interference and distortion in a signal.

In this paper we used MATLAB environment to generate the constellation diagram of M-ary PSK and QAM modulation systems.
\end{abstract}

Keywords: -Constellation, MATLAB, Modulation.

\section{INTRODUCTION}

By representing the transmitted symbol as a complex number and modulating a cosine and sine carrier signal with the real and imaginary parts (respectively), the symbol can be sent with two carriers on the same frequency. They are often referred to as quadrature carriers. A coherent detector is able to independently demodulate these carriers. This principle of using two independently modulated carriers is the foundation of quadrature modulation. In pure phase modulation, the phase of the modulating symbol is the phase of the carrier itself and this is the best representation of the modulated signal.

As the symbols are represented as complex numbers, they can be visualized as points on the complex plane. The real and imaginary axes are often called the in-phase, or I-axis, and the quadrature, or Qaxis, respectively. Plotting several symbols in a scatter diagram produces the constellation diagram. The points on a constellation diagram are called constellation points. They are a set of modulation symbols which compose the modulation technique.

Also a diagram of the ideal positions, signal space diagram, in a modulation scheme can be called a constellation diagram. In this sense the constellation is not a scatter diagram but a representation of the scheme itself. Upon reception of the signal, the demodulator examines the received symbol, which may have been corrupted by noise and InterSymbol Interference (ISI) in the channel or the receiver. If we represent noise and ISI on Euclidian space with power and time axis, then the noise internal and external, impulsive and induced, cross talk and attenuation are represented by power axis. Distortion, fading channel, phase distortion, delays, jitters and ISI are reflected by time axis [1].

For the purpose of analyzing the received signal quality, some types of corruption are very evident in the constellation diagram. For example:

- Gaussian noise shows as fuzzy constellation points

- Non-coherent single frequency interference shows as circular constellation points

- Phase noise shows as rotationally spreading constellation points

- Attenuation causes the corner points to move towards the center

A constellation diagram visualizes phenomena similar to those an eye pattern does for onedimensional signals. The eye pattern can be used to see timing jitter in one dimension of modulation. The instantaneous energy, or amplitude, of a symbol is its distance from the origin. The phase angle of a symbol is its angular displacement from the positive horizontal axis.

In literature, resent years, a number of research papers have been published on the constellation diagram of passband modulation techniques. In research paper [2] an expressions are derived for the evaluation of the bit error rate (BER) of binary phase shift keying (BPSK) and quadrature PSK (QPSK) signal constellations as a function of ADC word length (NADC) and signal to noise ratio (SNR) due to AWGN. It is shown that considerable performance gains can be achieved with an appropriate rotation of the signal constellation. Paper [3] shows the classification of digital techniques using constellation shape. In [4] Simulations have been performed for various SNR values and different types of QAM and PSK modulations. Channel model has been assumed to be an AWGN channel. Simulation results show that this method is very efficient and has high accuracy for the modulations recognition. The performances of this method for different types of QAM and PSK families have been investigated. Accuracy of this method for different modulations and 
different values of SNR is evaluated. The accuracy percentages have been obtained by executing the algorithm enough number of times and by calculating the ratio between correct recognition and total number of execution. The work [5] reports a new digital modulation recognition algorithm and proposed and implemented a technique that casts modulation recognition into shape recognition. This is possible by treating constellation shape as the key modulation signature. Recognition is also not limited to any specific class of modulations. In other words, if a modulation can be characterized by a constellation at all, it can be recognized. Although constellation reconstructions were shown for fading channels and other receiver imperfections, we have so far tested the algorithm in the presence of Gaussian noise alone.

\section{CONSTELLATION DIAGRAM OF BPSK}

BPSK have only two signal elements, one with a phase of $0^{\circ}$, and the other with a phase of $180^{\circ}$. BPSK also uses only an in-phase carrier. However, we use a polar NRZ signal for modulation. It creates two types of signal elements, one with amplitude (1) and the other with amplitude (-1) [6].

The MATLAB code and simulation results are shown in figure 1.
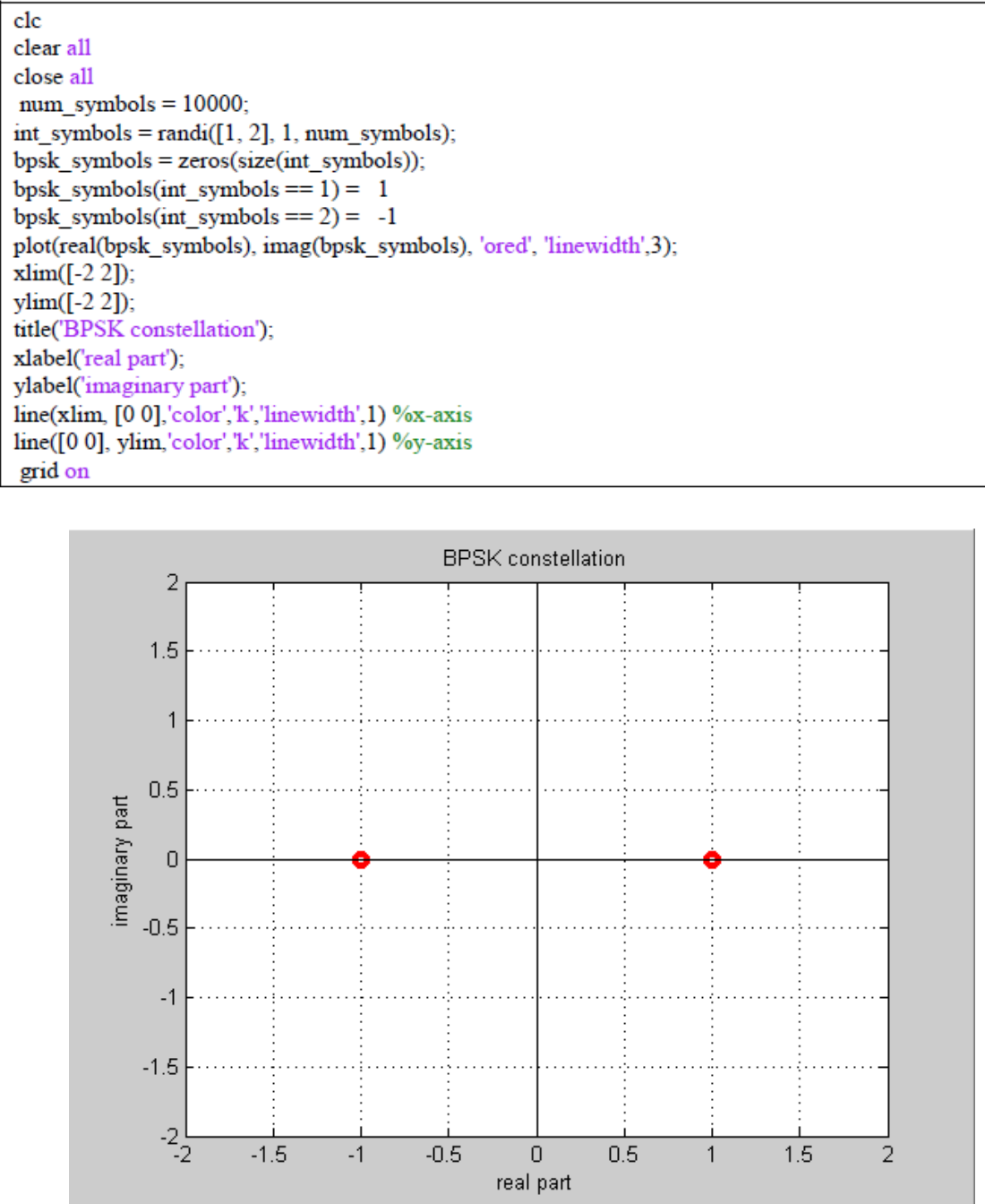

Fig. 1: Constellation diagram for BPSK in MATLAB.

\section{CONSTELLATION DIAGRAM OF QPSK}

QPSK uses four points on the constellation diagram, equally spaced around a circle. With four phases, QPSK can encode two bits per symbol. Constellation Diagram of QPSK also uses both an in-phase carrier and quadrature [7].

The developed MATLAB code for QPSK constellation diagram is shown below: 


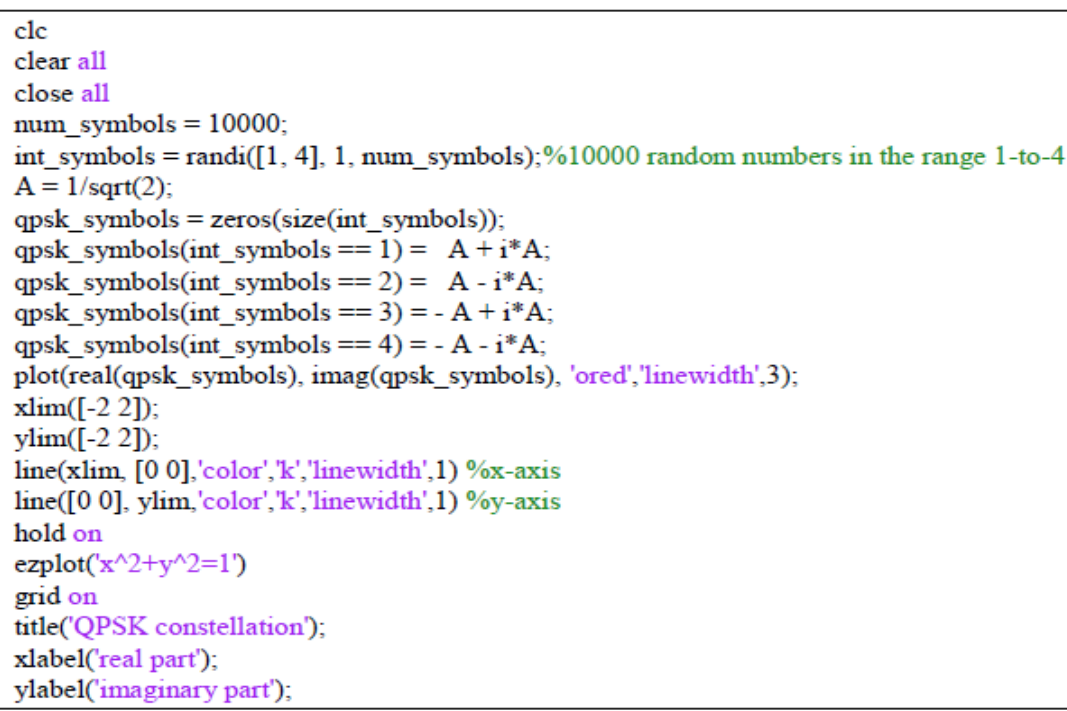

The simulation results are shown in figure 2 .

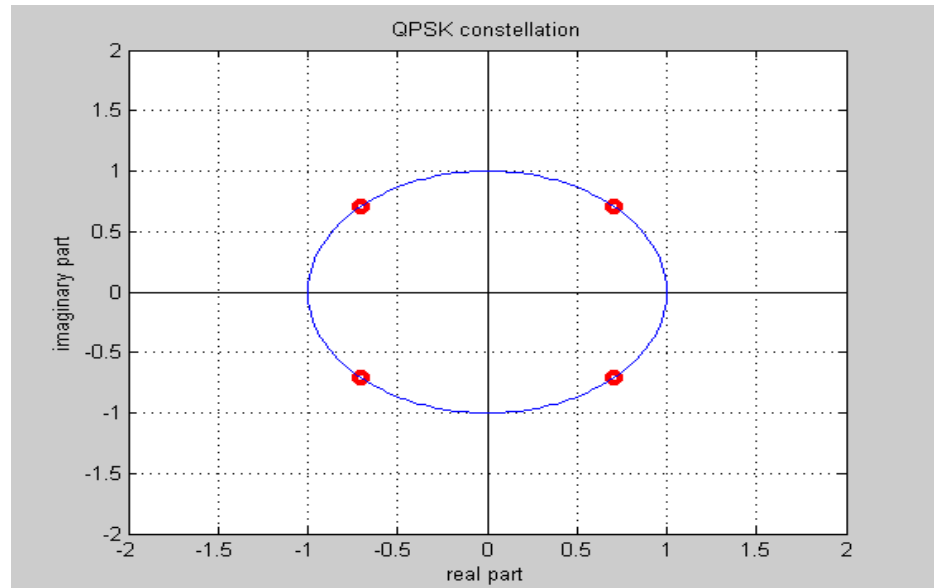

Fig. 2: Constellation diagram for QPSK in MATLAB.

In both result we note that the constellation diagram of QPSK use four phases that lead to more phase error and it would need a correlation receiver to discard the error phases.

A QPSK receiver must divide the signal constellation into decision regions, and then use these regions to identify the value of the received signal. For the constellation shown in Fig., the decision region boundaries are the real and imaginary axes. This makes the decision process very simple, since the receiver only needs to decide whether the signal magnitude is greater than zero or less than zero for both the in-phase and quadrature components. We could have chosen a symbol mapping that would rotate the constellation of Fig. 9 by $1 / 44$ radians, placing the symbols directly on the axes. However, in that case, the decision region boundaries would be half-way between the two axes, adding complexity to the receiver. Noise may cause the received signal to move out of the correct decision region. When this happens, the receiver will make an error in its symbol decision. Each symbol represents two bits; therefore, a symbol error may result in either one or two bits being in error. In order to minimize the bit error probability, we use a technique known as Grey mapping. Grey mapping requires symbols that are adjacent to each other in the signal constellation to differ by only one bit, as opposed to two bits. For each symbol in the constellation, the nearest neighbors will differ by only one bit, while the symbol furthest away will differ by two bits. This minimizes the bit error probability, since the probability of noise with large amplitude is less than the probability of noise with small amplitude.

\section{CONSTELLATION DIAGRAM OF 8-PSK AND 16-QAM}

Similarly to the above discussion, a MATLAB codes for 8-PSK and 16-QAM are modified and ran. The results are shown in figures 3 and 4 . 


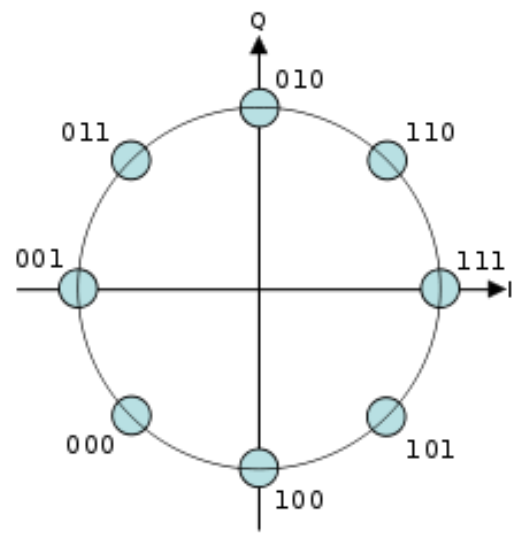

Fig. 3: Constellation diagram for Gray encoded 8-PSK.

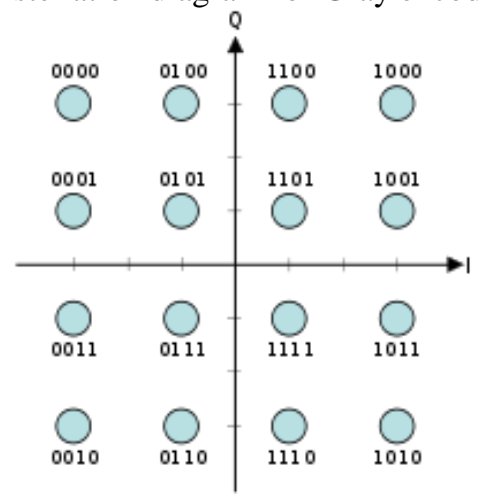

Fig. 4: Constellation diagram for rectangular 16-QAM.

\section{CONCLUSION}

From the simulation results shown in figures we can conclude that constellation diagram simplifies the analysis and comparison of digital passband modulation techniques. The developed MATLAB codes able to generate the constellation diagram for any M-ary modulation type which, in turn, makes the study of transmitting and receiving available and simplified.

\section{REFERENCES}

[1] Saed Thuneibat, Huthaifa Al Issa \& Abdallah Ijjeh "A Simplified Model of Bit Error Rate Calculation" Computer and Information Science; ISSN 1913-8989 E-ISSN 1913-8997, Published by Canadian Center of Science and Education, Vol. 9, No. 1; 2016, PP. 41-46.

[2] Umar H. Rizvi, Gerard J. M. Janssen; Jos H. Weber, "BER analysis of BPSK and QPSK constellations in the presence of ADC quantization noise", Browse Conference Publications, 2008, 14th Asia-Pacific Conference.

[3] B. Mobaseri; "Digital modulation Classification using constellation shape", Signal Processing pp. 251277, Jan 2000.

[4] Negar Ahmadi

[5] BijanG.Mobasseri"Digitalmodulationclassificationusingconstellationshape"https://www.researchgate.net/ publication/222076493, ArticleinSignal Processing, February 2000, DOI: 10.1016/S0165-1684(99)001279

[6] Kim, Kiseon, Polydoros; "Digital modulation recognition: the BPSK versus QPSK case", MILCOM '88, Oct. 1988, Vol. 2, pp.431-436.

[7] J. A. Sills, Maximum-likelihood modulation classification for PSK/'OAM, ILCOM '99, 